\title{
Contact eczema induced by hybrid manicure. The role of acrylates as a causative factor
}

\author{
Sandra Opalińska, Małgorzata Opalińska, Lidia Rudnicka, Joanna Czuwara
}

Department of Dermatology, Medical University of Warsaw, Warsaw, Poland

Adv Dermatol Allergol 2022; XXXIX (4): 768-774

DOI: https://doi.org/10.5114/ada.2021.109684

\begin{abstract}
Introduction: Acrylates are widespread plastic materials, known for their sensitizing properties. So far, allergy to acrylate monomers has been known as occupational eczema, mainly concerning dentists and manicurists. However, a surge of allergic contact dermatitis (ACD) cases related to acrylates among users of hybrid varnishes have recently been reported.

Aim: This article reviews the pathogenesis, clinical manifestations, and dermoscopic features of contact eczema induced by hybrid manicure.

Material and methods: The study was performed on a group of 8 women. Clinical and dermoscopic features were evaluated and correlated with the period of exposure to acrylates. In addition, all patients underwent mycological examination to exclude fungal co-infection.

Results: Mycological examinations in all patients gave negative results, although 1 patient developed local secondary mixed supra-infection due to Pseudomonas aeruginosa and Candida spp. Distribution of clinical manifestations corresponded to the area of contact with the allergen and comprised both skin and nail changes. The severity of inflammation correlated positively with the exposure period. Subungual hyperkeratosis and onycholysis were the most common findings ( $8 / 8$ patients), and eczematous finger pulp fissuring was a rarer sign ( $2 / 8$ patients) but more specific clinically.

Conclusions: The surge of contact dermatitis related to acrylates seen in recent years requires dermatologists' awareness. Nail changes induced by hybrid manicure can mimic onychomycosis or nail psoriasis. Therefore comprehensive patch testing should be performed in doubtful cases. Due to the lack of patch tests in our study, we can only suspect that we were dealing with allergic contact dermatitis. In case of confirmed allergy to acrylates, the patient should be aware of this and avoid them.
\end{abstract}

Key words: allergic contact dermatitis, acrylates, hybrid manicure.

\section{Introduction}

Acrylates are the polymerization products of acrylic and methacrylic acid under the influence of UV light [1] The correct polymerization process transforms the allergenic monomers into polymers, which are devoid of these properties. Acrylate monomers, despite their proven sensitizing properties, are still the main component of hybrid nail varnishes [2]. Because the sensitizing ability of monomers occurs when they are not completely polymerized, the increasing availability of UV lamps for domestic use has resulted in rapidly rising rates of allergic contact dermatitis (ACD) among their users. Non-professional low-power UV lamps prevent total polymerization of hybrid varnishes, leading to the development of an allergy to persistent unpolymerized monomers. Additionally, milling the outer layer of the nail plate and removing the cuticles before varnish application increases the penetration of the allergen into the nail bed.

Acrylates are also used in dental fillings, dentures, orthopaedic cement, contact lenses, hearing aids, glue for false eyelashes, and continuous glucose monitoring devices [1].

\section{Acrylates in styling and nail extension techniques}

The most frequently used methods in nail art include the following: applying tips, fiberglass technique, acrylic technique, light-curing gels use, hybrid, and titanium manicure. Acrylates are used in most of them. The use of

Address for correspondence: Joanna Czuwara MD, PhD, Department of Dermatology, Medical University of Warsaw, 82A Koszykowa St, 02-008 Warsaw, Poland, e-mail: joanna.czuwara@wum.edu.pl Received: 28.02.2021, accepted: 5.07.2021. 
tips is the simplest method, which utilises plastic plates that remain attached with an acrylate-based adhesive, usually ethyl cyanoacrylate [3]. The fiberglass technique consists of putting a piece of fiberglass or silk material on the nail and covering it with a special chemically cured resin, containing acrylic derivatives and hardening activator, also containing acrylates [3].

Acrylic nails arise as a result of mixing the liquid, which contains acrylic monomers, with powder containing acrylic polymers. Polymerization reaction takes place on the nail plate, resulting in the formation of an acrylic mass [4]. Before applying the acrylic mass, the nail is usually degreased with alcohols and other solvents. Then the so-called primer (most often methacrylic acid) is used on the natural plate. The task of the primer is to provide adhesion of the artificial nail [5].

The composition of UV gels is similar to that of acrylic nails, i.e. they contain acrylic monomers and an oligomer, a photo-initiator, and a light absorber [3]. The difference is that the polymerization reaction and hardening of the gel require UVA radiation, emitted by special lamps.

Hybrid varnish is applied similarly to traditional nail polish, with the difference that it dries only when it is cured with a UV or LED lamp. The whole process consists of polishing the nail plate, applying a hybrid base, a hybrid varnish, and then a hybrid top.

A powder manicure, or titanium manicure, is created by applying a special powder with an admixture of titanium and acrylic, which harden on the nail through contact with air [6]. It does not require the use of a UV lamp, and it seems to be the best alternative for women suffering from hybrid allergies.

\section{Aims}

This article presents and reviews the pathogenesis, clinical manifestations, and dermoscopic features of contact eczema induced by hybrid manicure, based on our experience in 8 patients.

\section{Material and methods}

This is a report of 8 women aged between 17 and 60 years with contact eczema related to acrylates found in hybrid varnishes. The exposure period ranged from 1 month to 3 years. In each case, the manicure was performed using a home acrylic nail kit equipped with a non-professional UV lamp. Furthermore, the majority of our patients had used varnishes from the same company. None of them had any nail diseases in the past; also, family history of psoriasis was negative in all subjects.

\section{Results}

Clinically, the majority of patients presented with hand eczema along with nail changes. Skin lesions were generally confined to the site of contact with the allergen, and the severity of inflammation depended on the time of exposure. The prominent symptom reported by patients was intense pruritus (8/8 women, $100 \%$ ). In more severe cases, pricking and pain were also present (4/8, 50\%). Skin examination revealed exfoliating erythematous and oedematous lesions localized on fingertips, especially in the periungual area (8/8, 100\%). In more severe cases $(2 / 8,25 \%)$ finger pulp erosions and extensive hyperkeratosis were observed. In all patients, the nail changes were induced by inflammation within the nail bed and nail matrix. The severity of inflammation was assessed by a score, in which (+) corresponded to dry exfoliation, $(++)$ to exfoliation with inflammation and erythema, and (+++) to oozing and bleeding (Table 1). The inflammatory process led to the onychodystrophy with subungual hyperkeratosis, resulting in nail plate elevation and onycholysis, which affected both its proximal and distal part. Nail dystrophy due to chronic periungual eczema was manifested as thickening and pitting of nails and transverse ridges. Dermoscopic examination enabled imaging of splinter haemorrhages, which probably arose secondary to the injury induced by hybrid manicure application. Other distinctive dermoscopic features beyond splinter haemorrhages included subungual hyperkeratosis leading to nail plate thickening, onycholysis with erythematous border surrounding the distal edge of the detachment, and distal nail plate splitting (Figure 1).

Such nail abnormalities strongly resemble psoriasis and mycosis. Therefore, each of our patients was directed initially to a mycological examination. Onychomycosis in all patients was excluded, although 1 patient developed local secondary bacterial and fungal super-infection due to chronic onycholysis. The causative organisms identified were Pseudomonas aeruginosa and Candida spp.

Exclusion of psoriasis was based on a negative family history, and accurate clinical and dermoscopic examination of the nails and nail-folds. The whole-body was evaluated for the presence of psoriasis. In doubtful cases biopsy of the nail fold or nail plate would have been an appropriate diagnostic method, but psoriasis was not suspected in any of our case.

Onycholysis and subungual hyperkeratosis were most often observed $(8 / 8,100 \%)$ (Tabe 1$)$. In severe cases, nail changes extended to the surrounding tissue and induced dermatitis of the fingertips (5/8, 62.5\%). Also, all 10 fingernails were affected. This indication can be a good clue in distinguishing these nail abnormalities from psoriatic and mycotic ones, in which nail changes are not so generalized as a rule. Also, patients with longer exposure to acrylates developed more severe changes, due to the fact that repeated expositions had caused a gradual amplification of the immune response and led to intensification of inflammation and skin changes after each exposure (Table 1). 
Table 1. The summary of representative pictures showing nails and nail-fold changes in all analysed patients. Clinical changes are described, and classification of the inflammation severity is given on the right side of the pictures. The scale according to which the severity of inflammation was assessed is marked as: (+) dry exfoliation, (++) exfoliation with inflammation and erythema, and (+++) oozing and bleeding. Also, the time of acrylates exposure is included

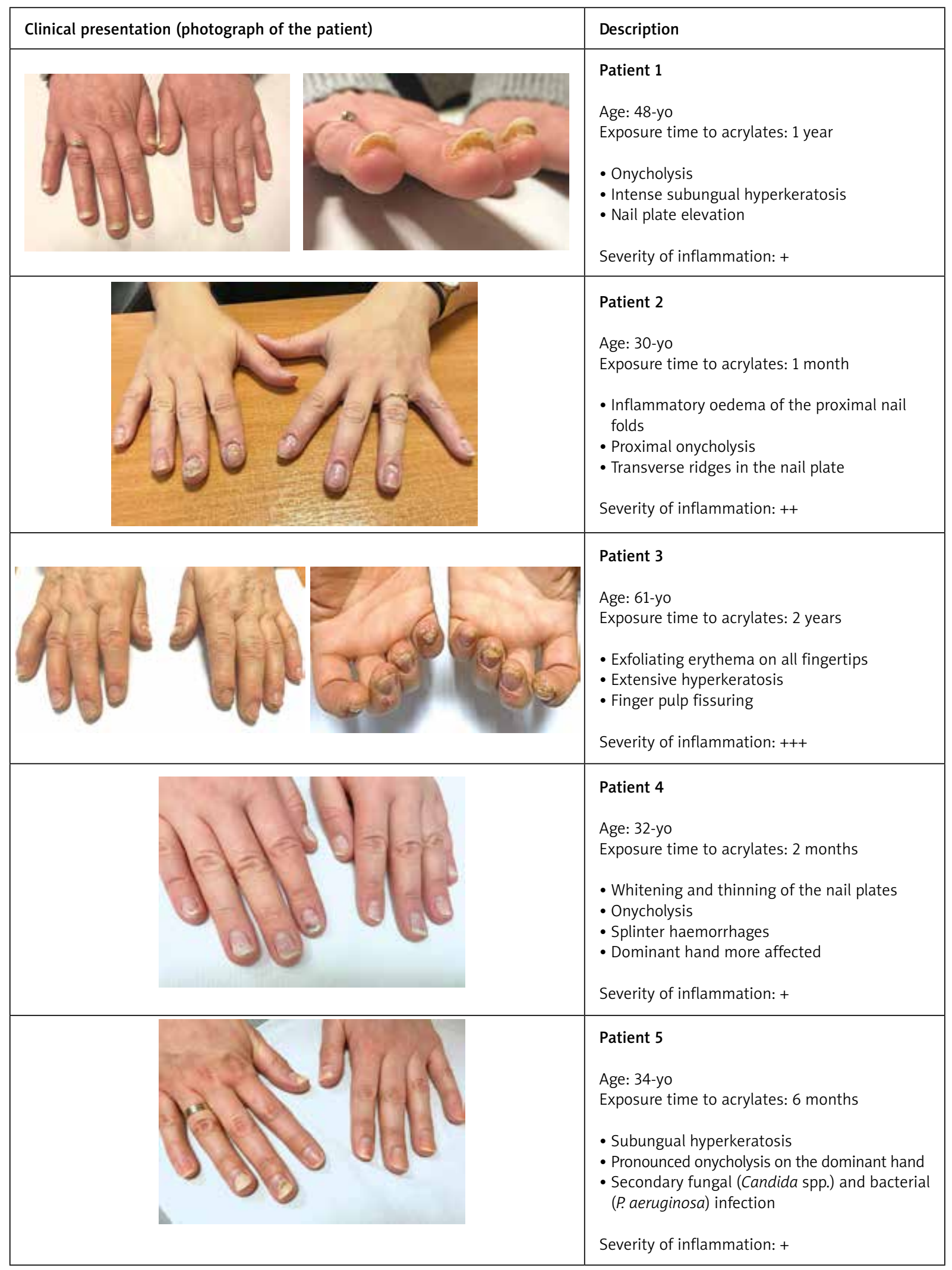


Table 1. Cont.

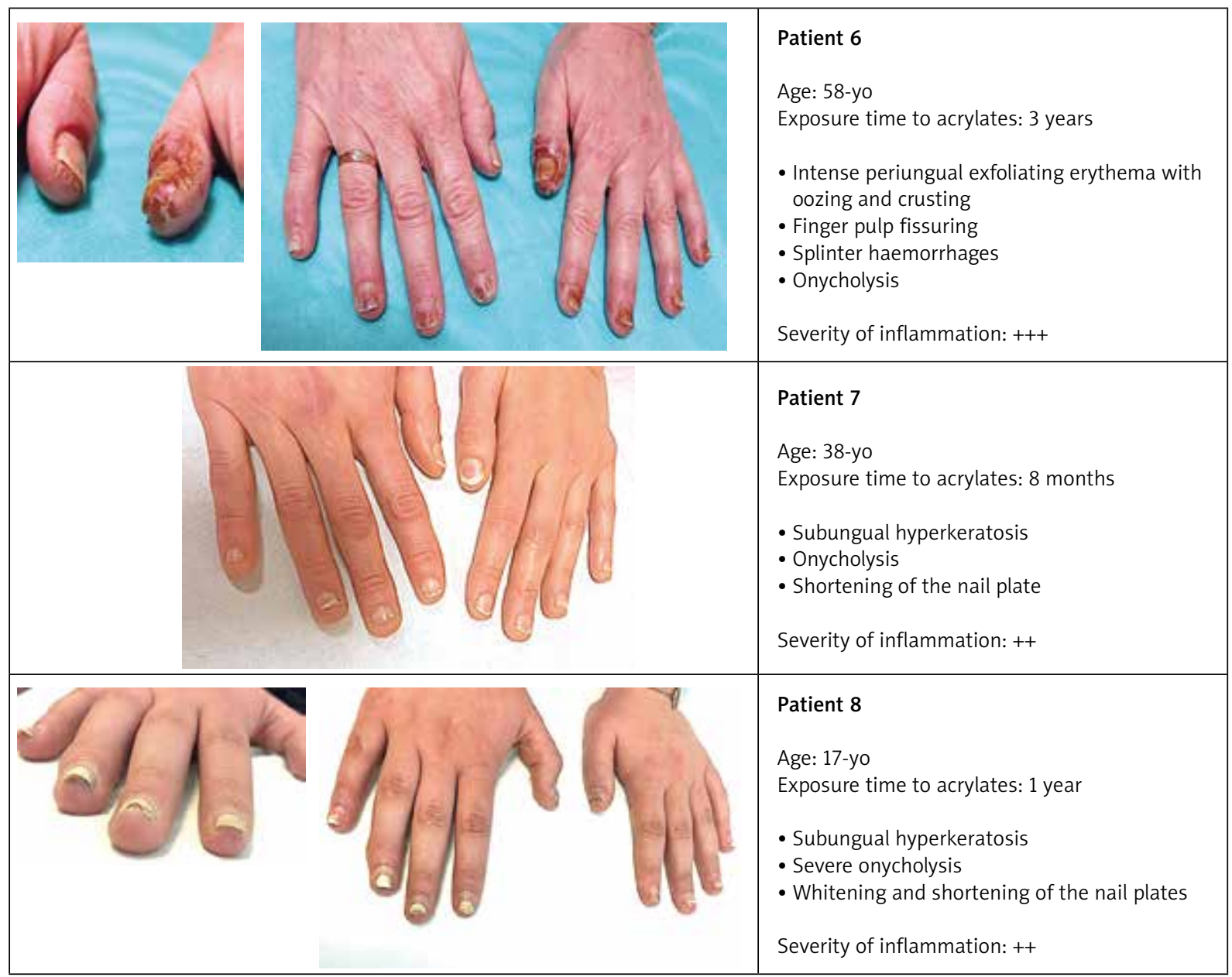

The diagnosis was based on a meticulous history taken in combination with a thorough physical examination and negative mycological results. Due to unavailability of patch tests in our study, we can only assume, based on the available literature, that it was allergic contact dermatitis. The treatment consisted of eliminating the trigger and hybrid manicure removal. Additionally, all patients were treated with combined topical medication, containing a potent corticosteroid (betamethasone), an antibiotic (gentamicin), and an antifungal medication (clotrimazole). Oral antihistamines were also used to quell the intense pruritus. After hybrid manicure removal with treatment, significant clinical improvement with resolution of inflammation was noticed, but post-inflammatory nail changes persisted for several months.

\section{Discussion}

The description of the above-mentioned patients indicates that new nail enhancement techniques can cause severe contact eczema. Such nail changes typically developed after months or even years of asymptomatic exposure to the allergen, so it can be assumed that this was a type IV allergic reaction (delayed hypersensitivity response). Pinpointing the proper cause in the majority of cases is usually delayed because people can expose themselves for years to the causative agent before their immune system responds to this chemical. For this reason, each case of dermatitis of the finger pulp with a history of hybrid manicure exposure should raise suspicion of contact allergy to acrylates. Moreover, in our experience, a titanium manicure can also cause severe contact eczema, although it seemed to be the best alternative for women suffering from hybrid allergies.

Simultaneously, it is worth remembering that inflammatory nail abnormalities induced by hybrid manicure, such as subungual hyperkeratosis or onycholysis, can mimic psoriasis or onychomycosis. Furthermore, histopathological analysis of the nail plate and subungual debris in both psoriasis and onychodystrophy secondary to acrylic nails can reveal neutrophils [7]. Due to the similarity of these diseases, in addition to mycological examination, comprehensive patch tests are required 

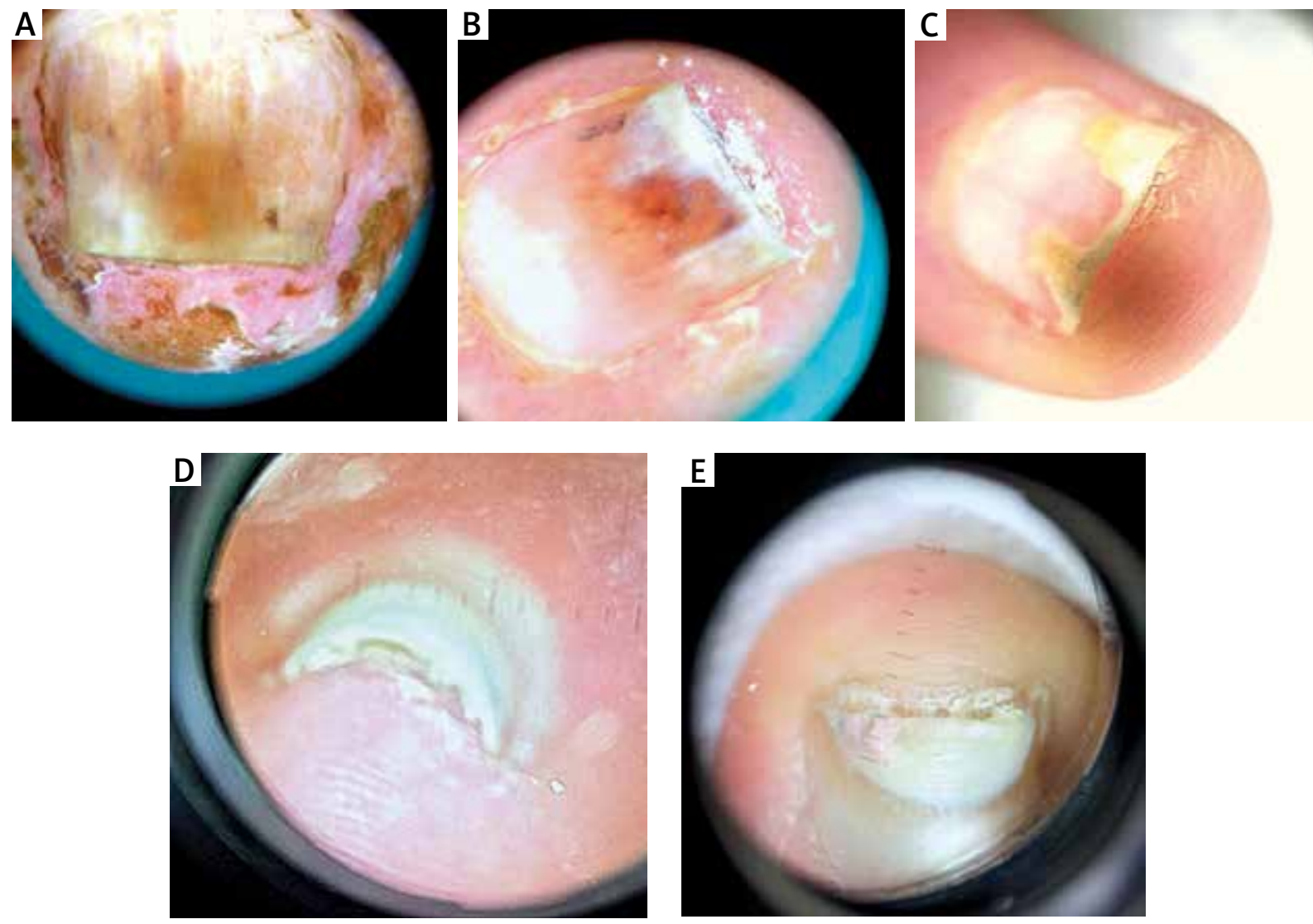

Figure 1. Dermoscopic examination revealed splinter haemorrhages, subungual hyperkeratosis with nail plate thickening, onycholysis with erythematous border surrounding the distal nail edge, and distal nail plate splitting

in ambiguous cases or a thorough clinical examination performed towards psoriatic skin changes. However, involvement of all nails and the absence of specific signs of nail psoriasis, such as pitting or oil spots, can suggest the correct diagnosis [8]. Moreover, it is worth noting that contact allergy usually causes a distal dystrophy corresponding to the area of contact with the allergen, and the nail matrix and proximal portion of the growing nail are unaffected [9]. Nevertheless, the use of topical antibiotics and antifungal medications in patients with allergic contact dermatitis is desirable because the presence of persistent onycholysis significantly increases the risk of secondary supra-infections, and glucocorticoid use intensifies such risk.

The chemical impact of acrylic nails and mechanical damage caused by nail filing can lead to brittleness and distal nail plate thinning, known as "worn-down nail syndrome" [10]. An electron microscopy study is also available, which showed that nails exposed to acrylic glue are rough and present a different morphology and composition in comparison to healthy human nails [11].

The surge of allergic contact dermatitis to acrylates seen in recent years is related to increased availability of home hybrid nail kits equipped with a UV light source.
A particularly high risk of sensitization among users is posed by UV lamps with a power below 36 watts, especially in the shape of bridges. Risk of sensitization and relapsing dermatitis may be mitigated by using appropriate light sources, completely curing the acrylic monomers and avoiding cutaneous exposure to uncured varnish [12].

Patch tests should be carried out to confirm allergic contact dermatitis. Unfortunately, in Poland the availability of patch tests for contact allergens in clinical practice is limited and acrylate allergens are available only for commercial use. Multiple studies have investigated the possibility of implementing a set of screening monomers to detect acrylate allergy [12]. Based on available literature, patch testing with 2-hydroxyethyl methacrylate (2HEMA) detects approximately $80 \%$ of acrylic allergy, and it may be considered as a good screening allergen [13]. The North American Contact Dermatitis Group currently includes ethyl acrylate (EA), methyl methacrylate (MMA), and 2-HEMA in its baseline series [12], and the European Society for Contact Dermatitis includes 2-HEMA [12]. Many recommendations have also appeared recently to include 2-HEMA into the British baseline patch test series [14]. However, no current consensus exists for an acrylate screening series due to the sensitivity and 
cross-reactivity of acrylic allergens and inconsistencies between studies [12]. It is usually necessary to perform patch tests on a broad range of acrylates to identify responsible compounds [12].

From an interdisciplinary point of view, acrylates may also induce cross-reactivity with other acrylic components used in dentistry, leading to allergic contact stomatitis [15] or orthopaedics and triggering severe allergic reactions when re-exposure occurs in a different location [16].

An analysis of the literature revealed the mounting reports of isobornyl acrylate (IBOA) allergy secondary to various medical devices, especially among patients with continuous glucose monitoring devices [17-20]. IBOA is an emerging source of acrylate allergy [12], which was named the "Contact Allergen of the Year" in 2020 by the American Contact Dermatitis Society [21].

In the early 1970s, the US Food and Drug Administration (FDA) banned the use of $100 \%$ liquid methyl methacrylate (MMA) in cosmetic products, but no regulation exists specifically to prohibit the use of MMA in lower concentrations [22]. MMA has been similarly restricted in the European Union, but is still widely available due to lack of enforcement and availability on-line [12]. Furthermore, the use of other methacrylate monomers is still allowed.

The Methacrylate Producers Association has also opined that ethyl (EMA) and methyl methacrylate (MMA) are not suitable for use in nail styling products due to the corrosive and sensitizing effect on the skin, but they are still being used as components in theirs products [23]. Moreover, no meaningful clinical data on exposure during pregnancy are available, but studies conducted in rats have proven that the dose of EMA and MMA affects the number of malformations in the offspring of the exposed females [24].

It is disturbing that new nail styling methods, as well as products used to make them, are not a subject of clinical trials. It needs to be highlighted that available clinical data in this topic come from case reports, and that products are banned solely on the basis of numerous user complaints, as has happened in Sweden [25]. To conclude, both public education and additional research studies referring to the safety of acrylic nail use are needed. What is more, it is necessary to update the regulations on mandatory labelling of medical devices as well as cosmetic products in order to prevent undesirable cross-reactions.

Limitations of the study include the small number of patients, and the lack of specific patch testing and histopathological examination due to the dermoscopic approach to the presented cases.

\section{Conflict of interest}

The authors declare no conflict of interest.

\section{References}

1. Sasseville D. Acrylates in contact dermatitis. Dermatitis 2012; 23: 6-16.

2. Arora H, Tosti A. Safety and efficacy of nail products. Cosmetics 2017; 4: 24.

3. Kieć-Świerczyńska M, Chomiczewska-Skóra D, ŚwierczyńskaMachura D, Kręcisz B. Manicurists and pedicurists - occupation group at high risk of work-related dermatoses. Med $\mathrm{Pr}$ 2013; 64: 579-91.

4. Constandt L, Hecke EV, Naeyaert JM, Goossens A. Screening for contact allergy to artificial nails. Contact Dermatitis 2005; 52: 73-7.

5. Baran R. Nail cosmetics. Am J Clin Dermatol 2002; 3: 547-55.

6. Metody manicure oraz środki stosowane w składzie płynów i proszków do manicure i ich wptyw na zdrowie. https:// manicuresystems.com/pl/substancje-chemiczne-w-manicure-kompendium-wiedzy.html.

7. Adigun CG, Shoaf H. Psoriasiform onychodystrophy induced by photobonded acrylic nails. J Clin Aesthet Dermatol 2020; 13: 18-20.

8. Mendonca MMS, LaSenna C, Tosti A. Severe onychodystrophy due to allergic contact dermatitis from acrylic nails. Ski Appendage Disord 2015; 1: 91-4.

9. Oppel T, Korting H. Onychodystrophy and its management. Ger Med Sci 2003; 1: Doc02.

10. Wu A, Morrison BW, Tosti A. Worn down nails after acrylic nail removal. Dermatol Online J 2015; 21: pii: 13030/qt5781m7vc.

11. Pinteala T, Chiriac AE, Rosca I, et al. Nail damage (severe onychodystrophy) induced by acrylate glue: scanning electron microscopy and energy dispersive X-ray investigations. Ski Appendage Disord 2017; 2: 137-42.

12. Voller L, Warshaw EM. Acrylates: new sources and new allergens. Clin Exp Dermatol 2019; 45: 277-83.

13. Ramos L, Cabral A, Gonçalo M. Allergic contact dermatitis caused by acrylates and methacrylates - a 7-year study. Contact Dermatitis 2014; 71: 102-7.

14. Rolls S, Chowdhury MM, Cooper S, et al. Recommendation to include hydroxyethyl (meth)acrylate in the British baseline patch test series. Br J Dermatol 2019; 181: 811-7.

15. Barber SK, Dhaliwal HK. Allergy to acrylate in composite in an orthodontic patient: a case report. J Orthod 2018; 45: 203-9.

16. Lazarov A. Sensitization to acrylates is a common adverse reaction to artificial fingernails. J Eur Acad Dermatol Venereol 2007; 21: 169-74.

17. Willingham E. Adhesive in acrylic nails, glucose monitors triggers allergy. Med Med News 28 $8^{\text {th }}$ EADV Congress, 2019. https://www.medscape.com/viewarticle/919558 (accessed Feb. 28, 2021).

18. Raison-Peyron N, Mowitz M, Bonardel N, et al. Allergic contact dermatitis caused by isobornyl acrylate in OmniPod, an innovative tubeless insulin pump. Contact Dermatitis 2018; 79: 76-80.

19. Herman A, de Montjoye L, Tromme I, et al. Allergic contact dermatitis caused by medical devices for diabetes patients: a review. Contact Dermatitis 2018; 79: 331-5.

20. Aerts O, Herman A, Mowitz M, et al. Isobornyl acrylate. Dermatitis 2020; 31: 4-12.

21. Militello M, Hu S, Laughter M, Dunnick C. American contact dermatitis society allergens of the year 2000 to 2020. Dermatol Clin 2020; 38: 309-20..

22. USF and D. Administration. Cosmetics Safety Q\&A: prohibited ingredients. FDA database. 2014. https://www.fda.gov/ 
cosmetics/cosmetic-products/nail-care-products (accessed Feb. 28, 2021).

23. I. Association. Methacrylate Producers. The methacrylate producers association's position on use of methacrylic and unreacted methacrylate monomers liquid form in artificial nail products. 2012. Available: http://static1.1.sqspcdn.com/ static/f/1405676/22020353/1361810987690/artificial nails2.pdf?token=RVAp9IhjikTwYatLr3EKnTSzkMU=.

24. Nicholas CA, Lawrence WH, Autian J. Embryotoxicity and fetotoxicity from maternal inhalation of methyl methacrylate monomer in rats. Toxicol Appl Pharmacol 1979; 50: 451-8.

25. Dahlin J, Berne B, Duner K, et al. Several cases of undesirable effects caused by methacrylate ultraviolet-curing nail Polish for non-professional use. Contact Dermatitis 2016; 75: 151-6. 